\title{
Die Nadelpincette, ein neues Instrument zur Pupillenbildung in starren Membranen
}

\author{
ron \\ Dr. Heymann.
}

Der fast erschöpfende Reichthum an Operationsmethoden im Gebiete der Augenheilkunde hat nur noch wenige Lücken im Bereiche der Heilmethoden jener Erblindungen übrig gelassen, welche bei vollkommner oder nahezu vollkommner Beschaffenheit der empfindenden Elemente ausschliesslich ihren Grund im Verschluss des Pupillengebietes für den Durchtritt der Lichtstrahlen haben. $\mathrm{Zu}$ den bisher oft noch unbesiegbaren, in der Regel aber wenigstens nur unvollständig zu beseitigenden Hindernissen dieser Art gehört vor Allem die Iritis mit Schwartenbildung, deren Wesen es ist, die Hinterfläche der Iris durch ein sehr steifes Gewebe neugebildeter Bindegewebsfasern nicht allein an und für sich in eine so straffe Membran zu verwandeln, dass das gewöhnliche Verfahren der Iridectomie wegen der Unmöglichkeit zwischen die Branchen der Pincette eine sich erhebende 
Falte zu bekommen, völlig aufzugeben ist, sondern auch eine feste Verwachsung zwischen Iris und vorderer Linsenkapsel oft im ganzen Gebiet ihrer Ausdehnung herzustellen. Wie schwierig es ist, gerade in Fällen der letzteren Art noch ein halbwegs befriedigendes Resultat zu erzielen, beweisen die vielseitig auf diesen Gegenstand gerichteten Versuche, welche noch bis in die neueste Zeit gemacht worden sind. Insbesondere hat der Gedanke an die Erfindung eines in solchen Fällen heilbringenden operativen Verfahrens unsern grossen uns leider so früh entrissenen von Gräfe noch bis in die letzte Periode seines reichen Lebens sichtlich beschäftigt, wie sein an die ophthalmologische Gesellschaft zu ihrer letzten Sitzung in Heidelberg, am 5. Sept. 1869 gerichtetes Telegramm beweist, in welchem er gegen die Hindernisse einer durch Schwartenbildung hinter der Iris verschlossenen Pupille in Fällen, wo schon die Linse vorher extrahirt war, die Iridectomie, sei es mit dem sichelförmigen Messerchen oder mit einem zweischneidigen myrthenblattförmigen Instrument auszuführen empfiehlt. Schon 9 Jahre vorher (Archiv VI.2 pag. 97) hatte er bei noch vorhandener Linse zur Erreichung eines Seherfolges die vorherige Extraction der meist noch ungetrübten Linse (damals ausdrücklich mittelst Lappenschnittes) empfohlen, welcher erst etwa 6 Wochen später die Pupillenbildung durch Einschlagen eines festen $\mathrm{Ha}$ kens in die Iris und ihre Schwarten, mit nachträglicher Entfernung von möglichst viel Masse derselben folgen sollte. Nach letzterem Verfahren hatte von Gräfe, nachdem er durch die ersten Erfolge desselben sehr ermuthigt worden war, eine grössere Anzahl von Fällen glücklich operirt; von letzterem Verfahren konnte er nur noch wenige Male, aber ebenfalls mit bestem Erfolge, Gebrauch machen. 
Aber nicht allein von Seiten der Iris werden solche starre Membranen gebildet, welche beim Aufegen der gewöhnlichen knieförmig gebogenen Pincette, keine Falte erheben lassen, sondern auch die Linsenkapsel gehört zu den Membranen dieser Art, welche, wenn sie in erheblicher Weise getrübt und dabei zugleich verdichtet ist, selbst mit dem Häkchen nicht hinreichend einzureissen ist, um ein für das Sehen genügend grosses Loch darin herzustellen. Diese Art Kapseltrübungen sind häufig die Folge einer selbst regelrecht ausgeführten Staarextraction, und ich stehe mit meinen Erfahrungen nicht vereinzelt da, wenn ich sie besouders bäufig nach der peripheren Linearextraction auftreten sah. Allerdings habe ich, wenn ich diese Behauptung aufstelle, eine grosse Zabl so feiner Kapseltrübungen im Sinne, dass sie nur mit seitlicher Focalbeleuchtung erkannt werden können, obgleich ihre Existenz dem Kranken schon erhebliches Nebelsehen und Verminderung der Sehschärfe verursachte. Man findet nichtselten, dass während der ersten Wochen nach der Extraction die Patienten sich der besten Sehschärfe erfreuen, die in der Regel nicht hinter $3 / 4$ zurückbleibt. Wenige Wochen später kehren diese Patienten wieder mit der Klage, dass sie nicht mehr so deutlich in die Ferne sehen, als früher. In der That bedürfen sie auch für die Nähe stärkerer Convexgläser, um so gut wie früher feine Schrift zu erkennen, und für die Ferme findet sich kein Glas, welches ihre Sehschärfe auf die früher vorhandene Höhe brächte. Vielen Kranken wird zwar durch cylind̛̀ische Gläser Verbesserung zu Theil und die werthvollen Beobachtungen von Reuss haben gewiss bei allen Augenärzten ungetheilten Beifall erhalten. Aber selbstverständlich ist von solchen reinen Brechungsanomalien nicht die Rede in den Fällen, welche ich hier im Sinne habe. Untersucht man das Auge genaver, um die Ursache der 
eingetretenen Sehschwäche, welche durch optische Hülfsmittel nicht zu heben ist, kennen zu lernen, so findet man bei seitlicher Focalbeleuchtung die Linsenkapsel von seidenartigem Glanz, ohne im Geringsten mit gröberen Trübungen behaftet $\mathrm{zu}$ sein. Bei genauerer Beobachtung des Kranken zeigt sich sodann, dass unter dem Einfluss von Licht und Luft bei der geringsten Steigerung desselben, die Umgebung der Hornhaut rosaroth injicirt wird, die Hornhaut selbst gewinnt einen vermehrten Glanz, das Auge zeigt sich von einer zarten Decke von Thränenflüssigkeit überzogen, die in sehr vielen Fällen nicht gross genug ist, um über die Lidränder abzulaufen, und darum oft völlig übersehen wird. Forscht man nach, ob der Kranke irgend eine Veränderung seines Sehens noch sonst beobachtet hat, so hört man häufig die Angabe, dass zuweilen weisse Wolken, mitunter auch flüchtige blitzartige Erscheinungen vor dem Auge aufgetreten seien. Ueber dunkle subjective Erscheinungen, in Form von Mouches volantes, wurde seltener geklagt. Bei sehr aufmerksamen Kranken wurde auch zuweilen ein ganz leiser Schmerz, gleichsam nur Druckgefühl, in der Stirn über dem afficirten Auge angegeben. Alle diese sowohl subjectiven, wie objectiven Symptome sind bei den in Rede stehenden Fällen von solcher Zartheit, dass sie sehr leicht vom Kranken and vom Arzt zu übersehen, oder falsch zu deuten sind. In Wirklichkeit. stellen sie aber die Symtomengruppe einer schleichenden Cyclitis und Capsulitis dar, durch welche sich die glasartige Linsenkapsel in eine Membran von mangelhaftiger Durchsichtigkeit und zugleich vermehrter Dichtigkeit verwandelt. Die Entfernung der getrübten, im durchfallenden Licht freilich oft noch ganz klar erscheinenden Linsenkapsel, im Bereich wenigstens einer mittelgrossen Pupillenöffnung giebt dem Kranken die volle Sehschärfe wieder, die er vorher besass, vorausgesetzt, dass durch 
geeignete übrige Behandlung der Irritationsprocess in der Kapsel sistirt wird. Wenn man aber eine solche Linsenkapsel, die sich der Natur der Sache nach, wenn überhaupt mit einer Pincette, so höchstens mit einer Häkchenpincette fassen lässt, mit einer solchen Pincette fast, so ist man über die Starrheit der Kapsel im Vergleich $z: x$ der nur so wenig gestörten Diaphanität oft überrascht. Auch solche Pincetten, welche ihre Häkchen im Niveau der auf der Kapsel aufzulegenden Fläche besitzen, genügen meist nicht, um ein wirkliches Fassen und Hervorziehen der resistenten Kapsel zu ermöglichen. Am nützlichsten erweist sich in solchen Fällen obne Zweifel das Häkchen, und insbesondere das Doppelhäkchen. Aber beide Instrumente, die ich oft angewendet, haben ihre Nachtheile. Yit dem einfachen Häkchen nämlich ist es entschieden Sache des Zufalls, ob man dadurch wirklich ein Stück Kapsel in Lappenform extrahirt, oder nur einen Schlitz in die Kapsel macht. Ich sage es ist Zufall, denn selbt die subtilst objective Untersuchung lässt uns über den Cohärenzgrad der seidenartig glänzenden Capsel im Stich und es erweist sich oft, was wir als weich und zart ansehen, in Wirklichkeit hart, selbst spröde. Das Doppelhäkchen aber, welches allerdings in jedem Falle eine breite Oeffnung herstellen muss, gewährt zunächst die bekannte manuelle Schwierigkeit des Oeffnens innerhalb des Auges, welches auch bei ruhigstem Manövriren nicht selten durch Störungen im Mechanismus des Instrumentes zu unerwarteten Bewegungen Veranlassung gibt; und sodann begegnen wir auch bei reinen Kapseltrübungen, besonders wenn verkalkte Linsenreste von der Kapsel noch eng umschlossen werden, nicht selten einer solchen Härte, dass die feinen Häkchen uns ihren Dienst versagen; ganz abgesehen davon, dass dieses feine Instrument den Schwarten, wie sie bei Iritis oft vorkommen, durchaus nicht gewachsen ist. 
Nach allem Diesem ist es wohl begreiflich, dass in Fällen der erwähnten Art jeder Operateur sich nach seiner Weise $\mathrm{zu}$ helfen suchte, und daher von einem allgemein genügenden Verfahren noch nicht die Rede sein kann. Ich habe die genannten Schwierigkeiten bis vor 2 Jahren in der oben erwähnten bisher gebräuchlichen Weise zu überwinden gesucht, und habe dabei manchen guten Erfolg gehabt. Gleichwohl erschien auch mir jedes der genannten Verfahren, wenn es günstig wirken sollte, so sehr an individuelle Eigenthümlichkeiten des Falles gebunden, dass es bei jedem einzelnen Falle räthlich erschien, sich auf die Nothwendigkeit mehrer derartiger Fingriffe zugleich gefasst zu machen. Insbesondere betraf dies die Frage, welches Hälschen einzuführen sei; denn nicht selten war es nöthig, nach nutzlosem Versuch mit dem gewöhnlichen zarten Irishäkchen zu einem stärkeren Haken überzugehen, mit welchem es trotzdem nur gelang, jene schlitzförmige Oeffnung hervorzubringen, aus welcher in der Regel Glaskörper in die vordere Augenkammer vordrang. In diese Spalte ging ich dann zumeist mit einer Pincette ein und suchte so viel als möglich von Iris, von Schwarte oder von Capsel zu fassen, um ein möglichst grosses Loch hervorzubringer.

Gerade solche Fälle nun brachten mich auf den Gedanken, den doppelten Eingriff durch Aenderung an den Instrumenten in einen einfachen $z \mathrm{u}$ verwandeln. Ich liess mir desshalb an einer kleinen $F$ ischer'schen Pincette die eine Branche vorn etwas verlän. gern und nadelförmigzuspitzen, so dass es nun möglich war, in jeden Falle die auch noch so starre Membran zu durchstechen und zugleich mittelst der anderen Branche in eine von der Stichöfnung entfernten Stelle der Membran einzuhaken. Mittelst kräftigen Schlusses der Pincette 
gelang es mir sodann bisher stets, auf einen Griff cine so breite Oeffnung herzustellen, dass ein nochmaliges Eingehen mit Instrumenten nicht nothwendig war. Wenn

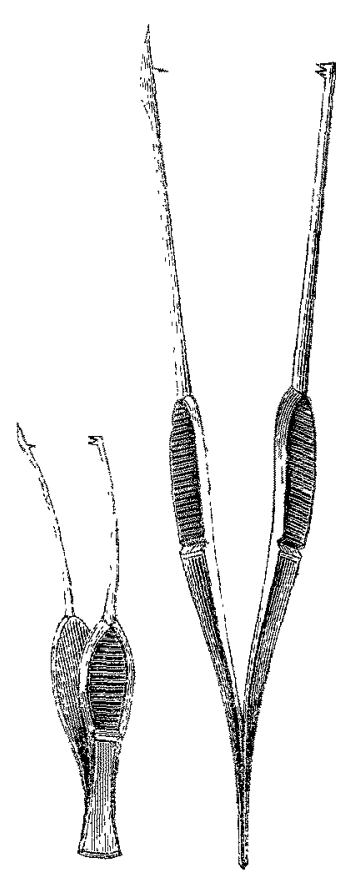

Natürliche Doppelte

Grösse. Grösse. ich mir erlaube, dieses kleine Instrument, das ich , Nadelpincette" zu nennen vorschlage, den Herren Collegen auf das Wärmste zu empfehlen, so bin ich dabei weit entfernt, dasselbe furr eine grosse Erfindung ausgeben zu wollen, darf aber wohl nach den zahlreichen glücklichen und leichten Erfolgen, die ich damit gehabt habe, dasselbe als eine empfehlenswerthe $\mathbf{E r}$ leichterung in Fällen von sonst oft excessiver Schwierigheit bezeichnen.

Was nun zunächst die Form des Instrumentes im Einzelnen anlangt, so ist die $\mathrm{F}$ ischersche Pincette, besonders mit ihren in einander. greifenden Zähnen an der Spitze zunächst vollständig dieselbe, wie sie sich in jedem augenärztlichen Operationsetui findet. Die eine Branche derselben läuft aber noch einige Millimeter weiter vor, als die andere, und dieser kurze Fortsatz überragt also die Gegend der Pincettenzähne. Ich habe demselben die Form einer breiteren Nadel mit doppelter Schneide und schlanker Spitze geben lassen, wobei die Breite der Nadel und ihrer Schneiden horizontal gestellt siucl. Auf diese Weise lässt sich die Pincette geschlossen durch jeden Linearschnitt einführen, ohne die 
Wundränder irgend wo zu verletzen. Zum Einstich genügt sodann eine ganz leichte Drehung der Pincette um ihre Längsaxe, so dass die Nadelbranche um Weniges tiefer steht, als die kürzere andere. In dieser Richturg gerade auf die Membran vorgeschoben muss die Nadel dieselbe durchstechen, während das Ende der küurzeren stumpfen Branche eben erst die Membran an ihrer Oberfläche berührt. Das Fassen mit der Pincette geschieht mun in der gewöhnlichen Weise, nur ist zu beachten, dass nicht beide Branchen dabei aneinander, sondern nur die stumpfe von der in die Membran schon eingesenkten Nadelbranche fortbewegt werden können. Will man daher der $z \mathbf{u}$ bildenden Oeffnung eine geiau beabsichtigte Grösse und Lage geben, so muss man die geschlossene Pincette nicht, wie sonst gebräuchlich, auf die Mitte der beabsichtigten Oeffnung zu schieben, sondern auf den äussersten Rand derselben. Wenn an dieser Stelle die Nadel durch die Membran bis zu ihrem Zähnchen eingedrungen ist, öfnet man erst die Pincette so weit, als es die beabsichtigte Breite der zu bildenden Oeffnung erfordert, wobei man zugleich die Pincette wieder in völlig horizontale Lage bringt. Schon wenn die stumpfe Branche mittelst ihres Häkchens irgend einen festen Punkt gewinnt, genügt es für das Zusammenfassen der zwischen beiden Branchen gelegenen Masse; denn in der Regel reisst dabei die Membran von der Einstichsöffnung aus leicht ein, und gestattet dadurch das gegenseitige Annäheru der Branchen. Mittelst Zug entfernt man sodann die Pincette sammt der gefassten Membran aus dem Innem des Auges. Hierbei allein tritt zuweilen eine geringe Schwierigkeit ein. Man muss nämlich die gefasste Membran mit der Nadelspitze etwas weiter hervorziehen, als mit einer gewöhnlichen, damit auch die Nadelspitze selbst aus der Wunde heraustritt. Diese zuweilen etwas starke Dehnung kann wohl zu einer 
Iriodialysis an der entgegengesetzten Seite führen. Indess ist mir in den 2 Jahren, wo ich das Instrument gebrauche und in mehr als 20 Fällen angewendet habe, dieser Vorfall noch nicht passirt. Noch weniger sah ich Reizung des corpus ciliare dadurch entstehen, welche bei einigermaassen derber Anwendung des Zuges wohl $z \mathfrak{u}$ fürchten wäre. In jedem Fall ist es nothwendig, um diese einzige Schwierigkeit zu verringern, die Nadel möglichst kurz anfertigen zu lassen, wodurch sie an Brauchbarkeit keineswegs verliert. Ueberdies muss ich bemerken, dass in den meisten Fällen die Membran ihrer pathologischen Beschaffenheit zu Folge in der Regel ausreisst, so dass man mit der Pincette ein völlig abgerissenes Stück sogleich entfernt, ohne erst mit der Scheere abschneiden zu müssen. Wenn aber Letzteres nöthig wird (sobald nämlich die Membran mehr dehnbar ist, und im Ganzen dem Zuge der Pincette folgt), so muss man die Pincette, sobald sie aus der Wunde hervorgezogen ist, seitlich, parallel zur Wunde, richten, damit die Scheere nicht durch die vorragende Nadelspitze am Schliessen verhindert wird.

Was nun die Fälle anlangt, in welchen das Instrament anwendbar ist, so eignen sich dazu zunächst alle aphakischen Augen, in welchen entweder ein fester Papillarverschluss mit straffgespannter Iris, oder eine gleichmässige, nicht lückenhafte Kapseltrübung vorhanden ist.

Beim festen Pupillenverschluss in Folge von Iritis nach der Linearextraction, halte ich das Instrument jedes Mal dann für anwendbar, wenn die Iris sich straff gespannt, und die, die Pupille verschliessende Membran völlig undurchscheinend zeigte. Bekanntlich ist es eine Eigenthümlichkeit der Iritis, nach peripherer Linearextraction, dass dabei die Pupille sich gegen die Wunde hin zumeist verengt, und daher in solchen Füllen, 
wenn der Schnitt von oben geführt wurde, die Pupille ganz nach oben verschoben, die gesammte untere Iris daher stark gedehnt erscheint. Wenn es in einem solchen Falle zur Bildung einer dichten Schwarte in der Pupille kommt, so ist anzunehmen, dass auch die Hinterfläche der Iris mit mehr weniger dichten Schwarten behaftet, und mit der Linsenkapsel locker oder fest verwachsen ist. Es bedarf zu dieser Annahme nicht erst der Zeichen der Atrophie an den Irisfasern, oder der stärkeren Vascularisation der Iris. Auch in solchen Fällen, wo das Parenchym der Iris noch wohlerhalten erscheint, lässt die Beschaffenheit der Pupillarmembran auf dichtere Massen hinter derselben schliessen. Man sticht daher bei so beschaffener Iris am besten mit der Nadelpincette ein und vollführt die Iridectomie im Uebrigen wie gewöhnlich; denn man ist dann sicher, eine Oeffnung bis zum Glaskörper zu erlangen, was bei der einfachen Iridectomie oft nicht der Fall ist. Einen Belag für die Richtigkeit dieser Ansicht gab mir folgender Fall:

Wilhelmine Grahl, $65 \mathrm{Jahr}$, wurde auf dem rechten Auge mittelst oberen peripheren Linearschnittes von einer reifen Cataract ohne jeden Zufall leicht befreit. Der sogleich nach der Operation angestellte Sehversuch ergab ein entsprechendes Sehresultat. Die Iris war nirgends in der Wunde eingelagert, die sehr weite colobomatische Pupille hatte ihren normalen Platz. Dem entsprechend verlief auch die Heilung binnen kurzer Frist ohne jegliche Reizerscheinungen, und am 4. Tage zeigte sich das Auge blass, nicht thränend, sehr gut sehend. Die Kranke hatte das Unglück, in der Nacht zum 5. Tage sich an das verbundene Auge, angeblick im Traume, heftig zu stossen. Am Morgen fand ich das Auge beim Oeffnen thränend, die Bindehaut der Sclera gleichmässig roth, die vordere Augenkammer mit Blut erfüllt, die Wunderänder ein wenig abstehend. Offenbar 
hatte bei dem Auge, welches übrigens ein Glotzauge war, der Stoss direct von vorn gewirkt und unter Abplattung der Hornhaut zur Spreizung der Wunde mit heftiger Zerrung der Iris geführt. Der anvermeidliche Verlauf war eine heftige Iritis, und nachdem das Blut der vorderen Augenkammer resorbirt worden war, zeigte sich beginnender Pupillarverschluss. Ein auch zu dieser Zeit noch bestehendes Unvermögen, selbst concentrirtes Licht wahrzunehmen, liess auf Glaskörperblutung mit Druck auf die Netzhaut schliessen, zu welcher Annakme die pralle Spannung des Bulbus Veranlassung gab. Im Verlauf mehrer Wochen verschwanden alle äussern Entzündungssymptome, die Spannung des Augapfels wurde normal, die Lichtempindung nahm sichtich zu. Endlich war der Zeitpunlt gelommen, wo es galt, eine neve Pupille zu bilden. Zu dieser Zeit war das Aeussere des Auges an allen Theilen normal, die Iris zeigte sich straff gespannt, übrigens aber nicht sichtbar verändert, gleichsarn gegen dio ganz nach oben gerückte Pupille geschoben, letztere war mit einer gelbweissen opaken Membran dicht verschlossen.

Ich machte einen Hornhautinearschnitt von unten, ging (damals) mit der gewöhulichen Irispincette ein, Konnte leicht eine breite Falte der Iris bilden und fassen, and excidirte dieselbe möglichst peripherisch. Eine lange, bis zum untern Hornhatrand reichende, und zasleich sehr breite Pupille war gebildet. Trotzdem gab lie Kranke nicht an, besser zu sehen, was ich zunächst auf einen rasch die Kammer anfüllenden Bluterguss schob. Als nach einigen Tagen die Untersuchung mit seitlicher Lokalbeleachtung gestattet war, zcigte sich de: Bluterguss völlig aufgesogen, der Augengrund aber durch eine dichte und allgemeine Kapseltrübung verdecht, wobei noch zahlreiche Pigmentstreifen und Haufen, die von der früher angeklebten Uvea herrührien, derselben auf- 
lagerten. Sobald es die Beschaffenheit des Auges gestattete, schritt ich zur Extraction der Capsel mit der Nadelpincette. In der That zeigte sich beim Einstich mit der Nadel die Capsel von einer solchen Resistenz, dass es mit einem Häkchen absolut unmöglich gewesen sein würde, dieselbe einzureissen. Der Nadelpincette folgte aber der gesammte centrale Theil der Capsel ohne Anwendung grosser Gewalt, indem der vordringende Glaskörper offenbar zum leichteren Einreissen der Membran beitrug. Das gefasste Stück riss sogleich ab, und es bedurfte keiner Scheere. Das entfernte Stück stellte eine pergamentartig gehärtete, sich aber leicht rollende, auf beiden Oberflächen rauh getrübte Capsel dar. Augenblicklich gab die Kranke an, jetzt ganz hell zu sehen. Der weitere Verlauf brachte Heilung ohne Zwischenfall.

Wie nun schon in diesem Falle, der ohne ausgeprägte Symptome sogenannter Schwartenbildung hinter der Iris auftrat, eine dichte Membran noch hinter der Iris gefunden wurde, welche zu einem zweiten Eingriff Veranlassung wurde, so ist mit noch weit mehr Sicherheit die Nothwendigkeit einer gewaltsamen Operation in Aussicht, wenn sich an der Iris die deutlichen Zeichen jener Entzündungsform zeigen, welche zu Schwartenbildung hinter der Iris zu führen pflegen. Wir schliessen bekanntlich auf das Vorhandensein letzterer, wenn die Oberfläche der Iris sich glatt ausgespannt, zugleich aber in ihren Fasern abgeplattet und zudem mit deutlich hervorragenden geschwellten Gefässen durchzogen zeigt. Diese Gefässe sind zumeist venös, deren Schwellung aus der gleichzeitigen Affektion der Ciliartheile resultirt, wodurch der venöse Rückfluss gehemmt ist. Man darf daher bei jeder. Operation an einer solchen Iris zuvor auf einen starken Bluterguss gefasst sein, und da der Bluterguss bei Iridectomien in der Regel zu einer allmählichen Verringerung des ursprünglichen Effektes 
fubrt, so ist in solchen Fällen die Excision gerade eines sehr grossen Theiles dringend geboten. Dieser Anforderung gegenüber stehen die bisherigen Operationsweisen entschieden machtlos. Die von y on Graefe hiergegen empfohlene Iridotomie setzt als Bedingung ihres Erfolges die noch mögliche Retractilität der Gewebe voraus. Von Graefe sagt*): „Die Gebilde sind meist (?) retraktil genug, um mit Hülfe des vorragenden und ausspreizenden Glaskörpers Schlitze zu bilden, welche Lichteinfall und Fortpflanzung des Glaskörperdrucks bis zur Hornhaut vermitteln." Ob aber diese Retractilität irgend woraus zu bestimmen ist, das scheint nach unseren bisherigen Erfahrungen fast unmöglich. Lin Bedenken gegen die Anwendung der Nadelpincette und dadurch erzengte Iridectomie könute vielleicht in dem Umstand liegen, dass bei solcher Schwartenbildung in der Regel starke Reaction mit erneuter Anbildung von Bindegewebsmassen stattfindet, wie auch v. Graefe a. a. 0 . anführt. Indess gilt gegenüber einer solchen Möglichkeit nur als Regel, dass man eine möglichst grosse Oeffnung mache, damit selbst bei eintretender Neubildung kein vollkommener Verschluss wieder eintrete. Die Grösse der Oeffnung hängt nun beim Gebrauch der Nadelpincette ganz von der Willkühr $a b$, und so babe ich denn auch in den Fällen von Schwartenbildung, wo ich mit dieser Pincette operirte, niemals nenen Verschluss, sondern höchstens eine Verkleinerung der ur* sprünglich angelegten Oeffnung gesehen. Es dürfte dies als Regel für den Verlauf solcher Operationen gelten, dass, je freiere Communication zwischen Glaskörper und Humor aqueus hergestellt wird, um so mehr Aussicht

* Klinische Monatsbläter fur Augenheilkunde VII. Nov. und Dez. pag. 432 . 
auf Bestand der einmal bewirkten Oeffnung vorhanden ist.

Somit scheint mir die Iridectomie mittelst Nadelpincette in Fällen von Iritis mit Schwartenbildung das allen andern entschieden vorzuziehende Verfahren zu sein.

Ich wende mich nun zunächst zur Behandlung der Kapseltrübungen nach derselben Methode.

Hier wirft sich vor Allem die Frage auf, ob die partielle Kapselextraction (denn eine solche wird in der That durch die Nadelpincette hervorgebracht) ein gefährlicherer Eingriff sei, als die Dilaceration mit der Nadel. Dass es nicht schwer ist, in Fällen von mässig harten oder weichen Kapseitrübungen mit der Nadel, oder noch besser nach Wilde mit 2 Nadeln eine genügende Oeffnung für den Lichteinfall herzustellen, darüber stimmen wohl alle Operateure überein. Aber es fragt sich einmal, ob die Dichtigkeit der vorhandenen Kapseltrübung a priori genau $z u$ bestimmen ist, und sodann, wenn diese Bestimmung sich als unsicher erweisen sollte, ob die Extraction weicher Kapseltrübungen bedenklicher ist, als ihre blosse Dilaceration.

In Bezug auf den letzten Punkt will ich vorausschicken, dass mich meine Erfahrungen nur in der Ansicht bestärkt haben, dass es jedes Mal sicherer ist, ein Kapselstück direct aus dem Auge zu entfernen, als es bloss zu dislociren. Im letzten Falle geschieht es nämlich nicht selten, dass der entstandene Zipfel, wo nicht völlig beweglich, doch nach und nach in seine frühere Lage zu gelangen im Stande ist. Was aber für mich noch mehr in die Wagschaale fällt, das ist die Umrandung der so geschaffenen Oeffnung. Mit der Dilacerationsmethode, selbst wenn sie mit zwei Nadeln ausgeführt wird, erhält man stets einen eckigen Defect in der Linsenkapsel, und ein solcher ist aus leicht begreiflichen 
Gründen leichter im Stande zu allmähliger Verengung, wo nicht Verschluss zu führen, als ein runder. Bei der Extraction, sie möge nur mit dem Häkchen, oder mit der von mir vorgeschlagenen Nadelpincette gemacht werden, entsteht jedesmal ein runder, oft auch oblonger Defect, und die Weite der entstandenen Deffnung ist um so viel grösser, dass auch ein bleibender Erfolg da. durch gesichert ist.

Dies sind die praktischen Gesichtspunkte, die mich von voruherein mehr für die Extraction geneigt machen. Es fragt sich nun, welche Gefahren die Methode, insbesondere mit der Nadelpincette hat. Es ist unleugbar, dass die Wirkung der Nadelpincette auf die getrübte Kapsel in mechanischer Rücksicht kein wesentlich anderer ist, als die des Häkchens, d. h. dass keine grössere Reaction durch die Nadelpincette daran hervorgerufen wird, als durch das Häkchen. Aber zum Einführen beider bedarf man zunächst eines linearen Schnittes, und in diesem könnte unter Umständen wohl eine Gefahr verborgen liegen. Da es sich hier in den weitaus meisten Fällen um Augen, die vorber am Staar operirt wurden, handelt (denn nur traumatische Cataracte entstehen mitunter ohne Verletzung der Sclera oder Hornhaut und geben nach erfolgter Pesorption dann zu gleicher Operation Veranlassung), so ist die Anlegung eines neuen Schnittes nicht immer gleichgültig. In dieser Beziehung habe ich aus eigener Erfahrung nur davor zu warnen, dass der neue Einschnitt zu nahe an den alten verlegt wird, daher vor sehr peripherisch dicht an der Scleralnarbe angelegten Hornhautschnitten. Hält man sich in grösserer Entfernung davon, oder schneidet man an einer entgegengesetzten Seite ein, so ist der Schnitt so unschuldig, wie jeder andere Linearschnitt. Bei zu nahe an der bestehenden Narbe angebrachten Horahautschnitten habe ich nochmals jene torpide Hornhautinfiltration 
auftreten gesehen, welche aus Ennährungsmangel eintritt und bekannt genug ist aus den Fällen traumatischer Keratitis, wo man neben dem entstandenen Leucom eine periphere Pupille bilden will. Dass der Schnitt in der Hornhaut, und zwar nicht einmal sehr nahe am Rande an. gelegt werde, balte ich deshalb für empfehlenswerth, weil die Wirkung der Nadelpincette, je steiler sie eingeführt werden kann, um so sicherer ist. 